\title{
Brugada syndrome risk loci seem protective against atrial fibrillation
}

\author{
Laura Andreasen $1,2,3$, Jonas B Nielsen ${ }^{1,2}$, Stine Darkner ${ }^{1,2}$, Ingrid E Christophersen ${ }^{1,2,4}$, Javad Jabbari ${ }^{1,2}$, \\ Lena Refsgaard $^{1,2}$, Jens J Thiis ${ }^{5}$, Ahmad Sajadieh ${ }^{6}$, Arnljot Tveit ${ }^{4}$, Stig Haunsø $\emptyset^{1,2,7}$, Jesper H Svendsen ${ }^{1,2,7}$, \\ Nicole Schmitt ${ }^{1,3}$ and Morten S Olesen ${ }^{\star, 1,2}$
}

Several studies have shown an overlap between genes involved in the pathophysiological mechanisms of atrial fibrillation (AF) and Brugada Syndrome (BrS). We investigated whether three single-nucleotide polymorphisms (SNPs) (rs11708996; G $>$ C located intronic to SCN5A, rs10428132; T > G located in SCN1OA, and rs9388451; T $>$ C located downstream to HEY2) at loci associated with BrS in a recent genome-wide association study (GWAS) also were associated with AF. A total of 657 patients diagnosed with AF and a control group comprising 741 individuals free of AF were included. The three SNPs were genotyped using TaqMan assays. The frequencies of risk alleles in the AF population and the control population were compared in two-by-two models. One variant, rs 10428132 at SCN1OA, was associated with a statistically significant decreased risk of AF (odds ratio $(O R)=0.77, P=0.001$ ). A meta-analysis was performed by enriching the control population with allele frequencies from controls in the recently published BrS GWAS (2230 alleles). In this meta-analysis, both rs10428132 at SCN1OA $\left(\mathrm{OR}=0.73, P=5.7 \times 10^{-6}\right)$ and $\mathrm{rs} 11708996$ at $S C N 5 A(O R=0.80, P=0.02)$ showed a statistically significant decreased risk of AF. When assessing the additive effect of the three loci, we found that the risk of AF decreased in a dose-responsive manner with increasing numbers of risk alleles ( $O R=0.50, P=0.001$ for individuals carrying $\geq 4$ risk alleles vs $\leq 1$ allele). In conclusion, the prevalence of three risk alleles previously associated with BrS was lower in AF patients than in patients free of $\mathrm{AF}$, suggesting a protective role of these loci in developing $\mathrm{AF}$.

European Journal of Human Genetics (2014) 22, 1357-1361; doi:10.1038/ejhg.2014.46; published online 26 March 2014

\section{INTRODUCTION}

Atrial fibrillation $(\mathrm{AF})$ is the most common arrhythmia that requires treatment and is associated with increased morbidity and mortality. ${ }^{1}$ The prevalence of AF increases with age, and affects $1-2 \%$ of the general population, a proportion that is likely to increase in the next 50 years. ${ }^{2,3}$ Cardiac risk factors such as hypertension and ischemic or other structural heart disease are known as risk factors for AF. ${ }^{1,4,5}$ However, a subgroup of patients diagnosed with AF is younger than 60 years of age and lacks these well-established risk factors. This condition is called 'lone' AF and accounts for $10-20 \%$ of the total number of patients with AF. 6 ,7

Brugada Syndrome $(\mathrm{BrS})$ is characterized by ST-segment elevations in the right precordial leads and an increased risk of ventricular arrhythmias and sudden cardiac death. ${ }^{8}$

There is a significant overlap between genes associated with $\mathrm{AF}$ and BrS. ${ }^{8,9}$ Accordingly, several rare variants in genes encoding cardiac sodium and potassium channels have been found both in patients with $\mathrm{AF}$ and in patients with $\mathrm{BrS}$. This is the case for the variants in SCN5A (c. 647C > T (p. (Ser216Leu)), rs201002736, ${ }^{10,11}$ c. $1127 \mathrm{G}>\mathrm{A}$ (p.(Arg376His)), rs199473101, ${ }^{10,12}$ c. $3157 \mathrm{G}>\mathrm{A}$ (p.(Glu1053Lys)), rs137854617,,$^{10,13}$ and c. $6010 \mathrm{~T}>\mathrm{C}\left(\mathrm{p} .\left(\right.\right.$ Phe2004Leu)), rs41311117 $\left.{ }^{14,15}\right)$, $S C N 1 B b$ (c. $641 \mathrm{G}>\mathrm{A}$ (p. (Arg214Gln)), rs66876876 $\left.{ }^{16,17}\right), \quad S C N 3 B$ (c. $29 \mathrm{~T}>\mathrm{C}$ (p.(Leu10Pro)), rs121918282 $\left.2^{18,19}\right)$, MOG1 (c. 181G $>$ T (p. $\left(\right.$ Glu61Xaa $\left.\left.{ }^{20,21}\right)\right), \quad$ rs140704891, and KCNJ8 (c. 1265C $>$ T (p.(Ser422Leu)), rs72554071 $1^{22,23}$ ). Furthermore, several studies have showed that the common AF-associated variant c. $1673 \mathrm{~A}>\mathrm{G}$ (p.(His558Arg)), rs1805124, in SCN5A may have a protective role against $\mathrm{BrS}$ in patients with other Brugada-associated SCN5A variants. ${ }^{11,24-26}$ Since some mutations can result in different phenotypes, ${ }^{18,19}$ the presence of additional single-nucleotide polymorphisms (SNPs) might have a disease-modifying effect. Of note, a recent genome-wide association study (GWAS) identified three loci (rs9388451; T > C, rs10428132; T > G, and rs11708996; $\mathrm{G}>\mathrm{C}$ ), to be associated with BrS. Patients carrying more than four risk alleles had an odds ratio (OR) of 21.5 for BrS, compared with patients carrying less than two risk alleles. ${ }^{27}$ All three GWASidentified loci are presumed to affect the sodium current.

Since $\sim 20 \%$ of Brugada patients also develop supraventricular arrhythmias including $\mathrm{AF}^{28}$ we hypothesized that SNPs previously associated with BrS in GWAS $^{27}$ may also modify the risk of AF.

\section{MATERIALS AND METHODS}

Study population

A total of 358 patients with lone AF (ie, the absence of clinical or echocardiographic findings indicating other cardiovascular diseases, metabolic, or pulmonary diseases) and onset of disease before the age of 50 (ranging from

${ }^{1}$ Danish National Research Foundation Centre for Cardiac Arrhythmia, Copenhagen, Denmark; ${ }^{2}$ Laboratory for Molecular Cardiology, Department of Cardiology, Rigshospitalet, University of Copenhagen, Copenhagen, Denmark; ${ }^{3}$ The Ion Channel Group, Department of Biomedical Sciences, University of Copenhagen, Copenhagen, Denmark; ${ }^{4}$ Department of Medical Research, Bærum Hospital, Vestre Viken Hospital Trust, Rud, Norway; ${ }^{5}$ Departments of Cardiothoracic Surgery and Cardiology, Rigshospitalet, University of Copenhagen, Copenhagen, Denmark; ${ }^{6}$ Department of Cardiology, Bispebjerg Hospital, University of Copenhagen, Copenhagen, Denmark; ${ }^{7}$ Department of Clinical Medicine, University of Copenhagen, Copenhagen, Denmark

${ }^{*}$ Correspondence: Dr MS Olesen, Laboratory for Molecular Cardiology, Department of Cardiology, Rigshospitalet, Juliane Mariesvej 9, 2100 Copenhagen $\varnothing$, Denmark. Tel: +45 35456506; Fax: +45 35456500; E-mail: morten.salling.olesen@rh.regionh.dk

Received 27 August 2013; revised 12 December 2013; accepted 26 December 2013; published online 26 March 2014 
16 to 49 years of age) were included from Copenhagen, Denmark and from Oslo and Vestre Viken, Norway, as described previously. ${ }^{29}$ In addition, 299 'non-lone' AF patients were included.

An ethnically matched control population was recruited from the same geographic area as the cases. This control population comprised (1) 208 healthy blood donors $<48$ years of age and free of cardiac diseases ${ }^{19}$ and (2) 533 men and women aged 55-75 years without history of AF or other cardiovascular diseases or stroke, despite a high prevalence of risk factors for $\mathrm{AF}^{30}$ (control group I). The latter control group was considered as highly relevant, because of their high AF resistance. To add power to our data, we added summarized data from the recently published BrS GWAS ${ }^{27}$ (control group II).

Written informed consent was obtained from all participants. The study conforms to the principles outlined in the Declaration of Helsinki, and was approved by the Scientific Ethics Committee of Copenhagen and Frederiksberg (Protocol reference number: H-KF-01313322) and the Regional Ethics Committee in Norway (Protocol reference number: 2009/2224-5).

\section{SNP genotyping}

Genotyping was performed as previously described. ${ }^{31}$ In brief, the DNA was extracted from whole blood that had been stored at $-20^{\circ} \mathrm{C}$ using the QIAamp DNA Blood Mini and Maxi kits (Qiagen, Hilden, Germany). SNP genotypes for rs9388451, rs10428132, and rs11708996 were determined using fluorescence-based real-time PCR (ABI PRISM 7900 Sequence Detection System; Applied Biosystems, Foster City, CA, USA) and pre-developed TaqMan assays (Applied Biosystems). An allelic discrimination run was performed allowing for discrimination between the allele compositions of each sample.

SNP genotypes for rs9388451, rs10428132, and rs11708996 have been submitted to ResearchGate, URL: https:/www.researchgate.net/publication/ 259990810_Brugada_syndrome_risk_loci_seem_protective_against_atrial_ fibrillation?ev=prf_pub.

Table 1 Clinical data of patient and control populations

\begin{tabular}{lccc}
\hline & Controls & Lone AF & Non-lone AF \\
\hline$N$ & 741 & 358 & 299 \\
Median age, years (IQR) & $60(55-70)$ & $35(28-39)$ & $61(51-69)$ \\
Male gender, \% & 52.3 & 81.9 & 69.5 \\
Hypertension, \%a & 26.2 & 0 & 36.5 \\
\hline
\end{tabular}

Abbreviations: $A F$, atrial fibrillation; IQR, interquartile range.

aHypertension defined as diastolic blood pressure $>90 \mathrm{~mm} \mathrm{Hg}$ or systolic blood pressure

$>140 \mathrm{~mm} \mathrm{Hg}$ at clinical examination.

\section{Statistical analyses}

The proportion of risk alleles (risk allele frequencies) was compared between $\mathrm{AF}$ cases and controls in two-by-two tables. Analyses were performed separately for the three investigated SNPs using either (1) in-house controls (control group I) or (2) both the in-house controls and summarized data on controls from the recently published BrS GWAS ${ }^{27}$ (control group I and control group II). The latter meta-analysis was done to add power to our analysis.

To assess the additive effect of the three SNPs, logistic regression models were performed including AF patients and control group I. Individuals were categorized into having $0-1,2-3$, or 4-6 risk alleles. The additive effect of carrying multiple BrS-risk alleles was assessed in four different models including (1) all three alleles, (2) the two loci that were statistically significant associated with $\mathrm{AF}$, (3) all three alleles but without the non-lone AF patients, and finally (4) only the two statistically significant loci and without the nonlone AF patients. A two-tailed $P$-value of $<0.05$ was considered as statistically significant. Analyses were performed with the Stata 11.0 software package (StataCorp LP, College Station, TX, USA).

\section{RESULTS}

Clinical characteristics of the two AF groups and the control group are shown in Table 1. The lone and non-lone AF groups are combined in one case group throughout all analyses, unless otherwise stated.

The results of the genotyping are summarized in Tables 2 and 3 . One of three variants, rs10428132 in SCN10A, showed a statistically significant decreased risk of $\mathrm{AF}(\mathrm{OR}=0.77, P=0.001)$ when comparing AF cases with control group I. Combining control groups I and II, both rs10428132 in SCN10A and rs11708996 intronic to SCN5A showed a statistically significant decreased risk of AF (rs10428132: $\mathrm{OR}=0.73, \quad P=5.7 \times 10^{-6}, \quad$ rs11708996: $\left.\quad \mathrm{OR}=0.80, \quad P=0.02\right)$ (Table 3).

In a pooled analysis of all three SNPs, we found that the risk of AF decreased consistently with increasing numbers of BrS-risk alleles (Figure 1a). Carrying four or more risk alleles was associated with an OR of 0.50 (95\% CI: $0.33-0.76, P=0.001)$ for AF compared with individuals carrying less than two risk alleles. A subgroup analysis of the lone AF patient group showed a similar association $(\mathrm{OR}=0.41$, 95\% CI: $0.23-0.74, P=0.003$ ) (Figure $1 b$ ). Similar pooled analyses were carried out for the two variants shown to be significantly associated with a decreased risk of AF. Again, AF risk decreased consistently with increasing numbers of BrS-risk alleles that an individual carried (Figure 1c and d). The distribution of risk alleles

Table 2 Effect size of BrS-risk alleles in AF population vs control I

\begin{tabular}{lcccccccc}
\hline Nearest gene & SNP & Chr & Bp substitution & Risk allele 27 & RAF cases & RAF control I & OR (95\% Cl) & P-value \\
\hline HEY2 & rs9388451 & 6 & C > T & C & 0.49 & 0.51 & $0.94(0.81-1.09)$ & 0.42 \\
SCN10A & rs10428132 & 3 & G $>$ T & T & 0.33 & 0.39 & $0.77(0.66-0.90)$ & 0.001 \\
SCN5A & rs11708996 & 3 & C $>$ G & C & 0.13 & 0.15 & $0.82(0.65-1.02)$ & 0.07 \\
\hline
\end{tabular}

Abbreviations: bp, basepair; Chr, chromosome; $\mathrm{Cl}$, confidence interval; OR, odds ratio; RAF, risk allele frequency; SNP, single-nucleotide polymorphism.

Control I: in-house controls. Cases: combined lone AF and non-lone AF patient group. P-value defined as two-sided.

Table 3 Effect size of BrS-risk alleles in AF population vs controls I+ II

\begin{tabular}{|c|c|c|c|c|c|c|c|c|c|c|}
\hline $\begin{array}{l}\text { Nearest } \\
\text { gene }\end{array}$ & SNP & Chr & $\begin{array}{c}B p \\
\text { substitution }\end{array}$ & $\begin{array}{c}\text { Risk } \\
\text { allele } 27\end{array}$ & $\begin{array}{l}\text { RAF } \\
\text { cases }\end{array}$ & $\begin{array}{c}\text { RAF control } \\
l\end{array}$ & $\begin{array}{c}\text { RAF control } \\
I^{27}\end{array}$ & $\begin{array}{c}\text { RAF controls } \\
I+I I\end{array}$ & $\begin{array}{c}\text { OR }(95 \% \mathrm{Cl}) \text { controls } \\
I+I I\end{array}$ & $\begin{array}{c}\text { P-value controls } \\
\qquad I+I I\end{array}$ \\
\hline HEY2 & rs9388451 & 6 & $\mathrm{C}>\mathrm{T}$ & C & 0.49 & 0.51 & 0.50 & 0.51 & $0.97(0.85-1.10)$ & 0.64 \\
\hline SCN1OA & rs10428132 & 3 & $\mathrm{G}>\mathrm{T}$ & $\mathrm{T}$ & 0.33 & 0.39 & 0.41 & 0.40 & $0.73(0.64-0.84)$ & $5.7 \times 10^{-6}$ \\
\hline$S C N 5 A$ & rs11708996 & 3 & $C>G$ & C & 0.13 & 0.15 & 0.15 & 0.15 & $0.80(0.66-0.98)$ & 0.02 \\
\hline
\end{tabular}

Abbreviations: bp, basepair; Chr, chromosome; Cl, confidence interval; OR, odds ratio; RAF, risk allele frequency; SNP, single-nucleotide polymorphism.

Abbreviations: bp, basepair; Chr, chromosome; Cl, confidence interval; OR, odds ratio; RAF, risk allele frequency; SNP, single-nucleotide polymorphism.
Control II: GWAS ${ }^{27}$ controls, controls I + II: in-house + GWAS ${ }^{27}$ controls. Cases: combined lone AF and non-lone AF patient group. $P$-value defined as two-sided. 

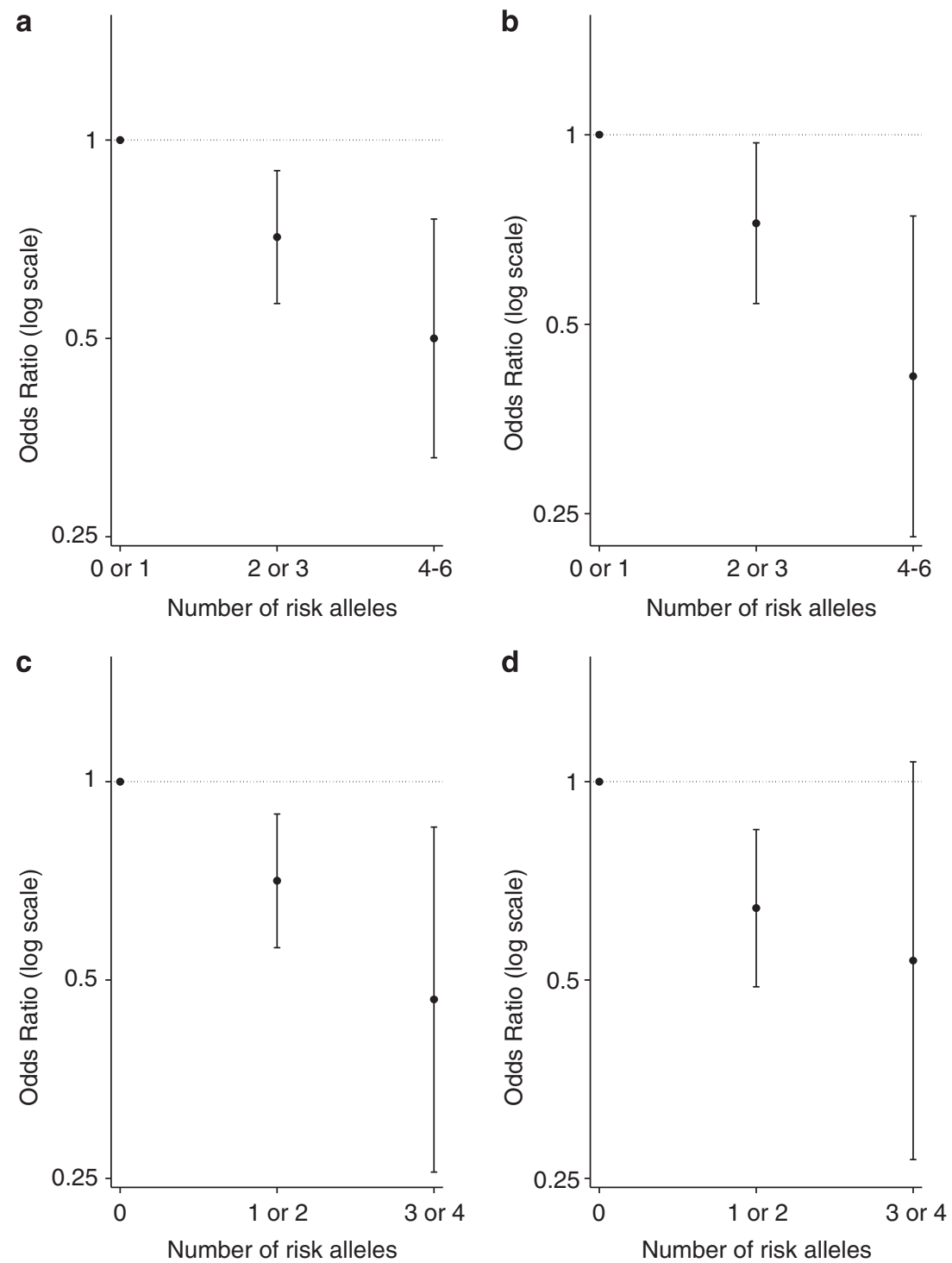

Figure 1 ORs calculated according to the number of risk alleles in the in-house controls. Each dot represents the $\log (\mathrm{OR})$ value and each bar the $95 \%$ confidence interval (vertical). (a) Pooled analysis of all three loci in the lone AF and the non-lone AF patients vs the in-house controls. (b) Pooled analysis of all three loci in the lone AF patients vs the in-house controls. (c) Pooled analysis of the two loci significantly associated with AF (rs10428132 in SCN1OA and rs11708996 intronic to SCN5A) in the lone AF and the non-lone AF patients vs the in-house controls. (d) Pooled analysis of the two loci significantly associated with AF in the lone AF population vs the in-house controls.

in patients and controls (control group I) is shown in Figure 2. The risk of AF decreases consistently with increasing numbers of BrS-risk alleles, indicated by the right-shifted patient population.

\section{DISCUSSION}

We investigated the prevalence of three SNPs recently shown to be associated with $\mathrm{BrS}$, in a group of $\mathrm{AF}$ patients and in controls, and found that the additive effect of the three loci decreased the risk of AF in a dose-response manner $(\mathrm{OR}=0.50,95 \% \mathrm{CI}$ : $0.33-0.76, P=0.001$ in the presence of $\geq 4$ risk alleles $v s \leq 1$ ).

The finding that the same group of SNPs known to be associated with $\mathrm{BrS}$, with ORs of $>20$, at the same time possess a protective effect against AF is fascinating. The reported SNPs are either within or close to genes underlying or regulating the cardiac sodium current $\left(I_{\mathrm{Na}}\right)$. rs10428132 resides in the gene SCN10A encoding $\mathrm{Na}_{\mathrm{V}} 1.8$, rs11708996 is intronic to SCN5A encoding $\mathrm{Na}_{\mathrm{V}} 1.5$, and rs9388451 resides near the HEY2 locus. The loci at SCN10A and SCN5A are also associated with both PR interval and QRS duration. ${ }^{32,33}$

From an evolutionary perspective, our results may suggest that these common variants known to increase the risk of a rare, but potentially lethal syndrome such as BrS seem to have a dualistic effect, protecting against a much more common disease such as AF.

Noteworthy, the SCN10A SNP, rs10428132, is in high linkage (0.98 $r^{2}$ ) with rs6795970, also located in SCN10A. rs6795970 has, in a recent GWAS, ${ }^{34}$ been shown to protect against $A F$ and atrial flutter $\left(\mathrm{OR}=0.85, P=8.45 \times 10^{-4}\right)$, along with arterial embolism and thrombosis $\left(\mathrm{OR}=0.69, P=3.20 \times 10^{-3}\right)$. This SNP leads to the substitution of valine to alanine at amino-acid position 1073 (c. 3218T >C (p.(Val1073Ala))) in the sodium channel isoform Nav1.8, resulting in this channel displaying both increased peak and late sodium current in functional studies. ${ }^{35}$ The $\mathrm{Na}_{\mathrm{V}} 1.8$ channel protein seems to be expressed in working myocardium and the cardiac 


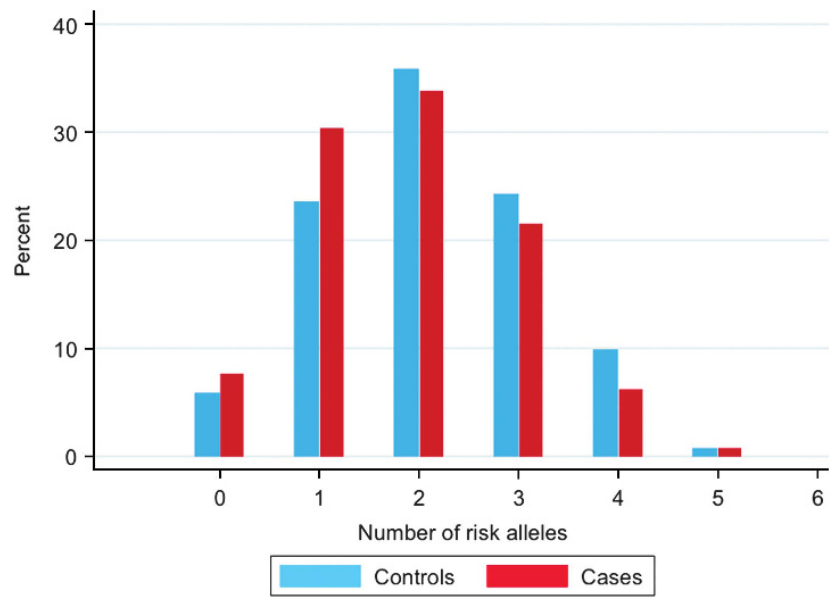

Figure 2 Distribution of risk alleles among individuals with AF (red bars) and among the in-house controls (blue bars).

conduction system, ${ }^{36}$ although it has also been found in intracardiac neurons. ${ }^{37}$ Its contribution to the total cardiac sodium current in the atria remains elusive. In addition, rs10428132 is in complete linkage with the PR-prolonging allele of rs6800541 which Pfeufer et $a^{32}$ also noted to have a protective effect on AF risk. Finally, rs10428132 is in almost complete linkage with rs6801957 $\left(r^{2}=0.97\right)$, which has been reported to affect the binding of the $T B X 3$ transcription factor and thereby the regulation of SCN5A/SCN10A expression. ${ }^{38}$

The majority of the cardiac sodium current is conducted by $\mathrm{Na}_{\mathrm{V}} 1.5$, and several studies including knock-out mouse models suggest a central role of $S C N 5 A / \mathrm{Na}_{\mathrm{V}} 1.5$ in the pathogenesis of AF. Heterozygote knock-out mice display compromised conduction velocity, impaired AV conduction, and QRS prolongation. ${ }^{39}$ An altered SCN5A/SCN10A expression could affect the conduction velocity and thereby the risk for BrS. Of note, both the SCN5A SNP rs11708996 and the SCN10A SNP rs10428132 have previously been associated with PR interval duration in a GWAS, ${ }^{32}$ supporting a protective role in AF. Pazoki et $a l^{40}$ replicated the association between the SCN5A SNP and PR duration, showing a trend for association between the rs11708996 $\mathrm{C}$ allele and a PR interval of $\geq 200 \mathrm{~ms}$ ( $\mathrm{OR}=2.39, P=0.004)$.

rs9388451 is located in close proximity to the HEY2 gene, encoding a transcriptional repressor important in the development of the cardiovascular system. ${ }^{41-44}$ Bezzina et al ${ }^{27}$ found homozygous HEY2 knock-out embryos to have increased $\mathrm{Na}_{\mathrm{V}} 1.5$ expression and a flattened transmural expression gradient of the channel in the cardiac ventricles. In $H E Y 2+1-$ knock-out mice, the conduction velocity was increased in the right ventricle outflow tract (RVOT), and the action potential upstroke velocity was increased, indicating an increase in sodium channel peak current. This is in line with the characteristic ST-segment elevation in the right precordial leads of BrS ECGs, and proposes the RVOT as an origin of ventricular arrhythmias in BrS. Assuming that the variant also gives rise to an increased conduction velocity in the atria it may protect against other genetic variants, or age- and sport-induced decreases in conduction velocity caused by fibrosis, and thereby protect against AF. Furthermore, HEY2-deficient embryos displayed a change in the expression patterns of GJA5, NPPA, and TBX5. ${ }^{43,45,46}$ These genes have all previously been associated with $\mathrm{AF}^{47-50}$

Thus, several studies support our finding that BrS-associated genetic variants protect against AF. Further mechanistic studies might help decipher how cellular electrophysiological changes induced by each of these variants (separately and additively) result in protection against AF. The elucidation of the mechanism(s) might provide keys to a more detailed understanding of the pathophysiology of AF and BrS. From a clinical point of view, our result highlights the complexity of personalized medicine as the AF patients with BrS-risk alleles, known to increase the sodium peak current, on one hand might benefit from a sodium peak current blocker such as flecainide, but on the other hand, flecainide treatment might increase the risk of sudden cardiac death in these patients, since they are predisposed for BrS by the same BrS-risk alleles.

\section{Study limitations}

Our study population was highly selected and relatively small. As a consequence, the power to detect associations was reduced. The study only included cases of Scandinavian ancestry, and the generalizability of the results to other ethnicities might be limited.

\section{CONCLUSIONS}

We found the risk of AF to decrease consistently with increasing numbers of BrS-risk alleles carried, suggesting that these variants have a protective role against developing AF.

\section{CONFLICT OF INTEREST}

The authors declare no conflict of interest.

\section{ACKNOWLEDGEMENTS}

We are grateful to the cardiology departments at Frederiksberg, Hvidovre, Gentofte, Herlev, Glostrup, Rigshospitalet, Amager, and Bispebjerg, and the Department of Medical Research at Bærum Hospital, Norway, for their help with the inclusion of patients. This work was funded by a framework grant of the Danish National Research Foundation, a grant of the Danish Council for Independent Research Medical Sciences, The John and Birthe Meyer Foundation, The Arvid Nilsson Foundation, Director Ib Henriksens Foundation and Fondsbørsvekselerer Henry Hansen og Hustru Karla Hansen, født Westergaards, legat.

1 Benjamin EJ, Wolf PA, D’Agostino RB, Silbershatz H, Kannel WB, Levy D: Impact of atrial fibrillation on the risk of death: the Framingham Heart Study. Circulation 1998; 98: 946-952.

2 Camm AJ, Kirchhof P, Lip GY et al: Guidelines for the management of atrial fibrillation: the Task Force for the Management of Atrial Fibrillation of the European Society of Cardiology (ESC). Eur Eur Pacing Arrhythm Card Electrophysiol J Work. Groups Card Pacing Arrhythm Card Cell Electrophysiol Eur Soc Cardiol 2010; 12 1360-1420.

3 Go AS, Hylek EM, Phillips KA et al: Prevalence of diagnosed atrial fibrillation in adults national implications for rhythm management and stroke prevention: the AnTic oagulation and Risk Factors in Atrial Fibrillation (ATRIA) Study. JAMA 2001; 285: 2370-2375.

4 Krahn AD, Manfreda J, Tate RB, Mathewson FA, Cuddy TE: The natural history of atrial fibrillation: incidence, risk factors, and prognosis in the Manitoba Follow-Up Study. Am J Med 1995; 98: 476-484.

5 Psaty BM, Manolio TA, Kuller LH et al: Incidence of and risk factors for atrial fibrillation in older adults. Circulation 1997; 96: 2455-2461.

6 Healey JS, Parkash R, Pollak T, Tsang T, Dorian P: Canadian Cardiovascular Society atrial fibrillation guidelines 2010: etiology and initial investigations. Can J Cardiol 2011; 27: 31-37

7 Fuster V, Rydén LE, Cannom DS et al: 2011 ACCF/AHA/HRS focused updates incorporated into the ACC/AHA/ESC 2006 Guidelines for the management of patients with atrial fibrillation: a report of the American College of Cardiology Foundation/ American Heart Association Task Force on Practice Guidelines developed in partnership with the European Society of Cardiology and in collaboration with the European Heart Rhythm Association and the Heart Rhythm Society. J Am Coll Cardiol 2011; 57: e101-e198.

8 Nielsen MW, Holst AG, Olesen S-P, Olesen MS: The genetic component of Brugada Syndrome. Front Card Electrophysiol 2013; 4: 179.

9 Olesen MS, Nielsen MW, Hauns $\varnothing$ S, Svendsen JH: Atrial fibrillation: the role of common and rare genetic variants. Eur J Hum Genet 2013; 22: 297-306. 
10 Darbar D, Kannankeril PJ, Donahue BS et al: Cardiac Sodium Channel (SCN5A) variants associated with atrial fibrillation. Circulation 2008; 117: 1927-1935.

11 Marangoni S, Resta CD, Rocchetti M et al: A Brugada syndrome mutation (p.S216L) and its modulation by p.H558R polymorphism: standard and dynamic characterization. Cardiovasc Res 2011; 91: 606-616.

12 Rossenbacker T, Carroll SJ, Liu H et al: Novel pore mutation in SCN5A manifests as a spectrum of phenotypes ranging from atrial flutter, conduction disease, and Brugada syndrome to sudden cardiac death. Heart Rhythm 2004; 1: 610-615.

13 Priori SG, Napolitano C, Gasparini M et al: Natural history of Brugada syndrome insights for risk stratification and management. Circulation 2002; 105: 1342-1347.

14 Bébarová M, O'Hara T, JL Geelen et al: Subepicardial phase 0 block and discontinuous transmural conduction underlie right precordial ST-segment elevation by a SCN5A loss-of-function mutation. Am J Physiol Heart Circ Physiol 2008; 295: H48-H58.

15 Olesen MS, Yuan L, Liang B et al: High prevalence of long QT syndrome-associated SCN5A variants in patients with early-onset lone atrial fibrillation. Circ Cardiovasc Genet 2012; 5: 450-459.

16 Olesen MS, Holst AG, Svendsen JH, Hauns $\varnothing$ S, Tfelt-Hansen J: SCN1Bb R214Q found in 3 patients: 1 with Brugada syndrome and 2 with lone atrial fibrillation. Heart Rhythm 2012; 9: 770-773.

$17 \mathrm{Hu}$ D, Barajas-Martínez $\mathrm{H}$, Medeiros-Domingo A et al: A novel rare variant in SCN1Bb linked to Brugada syndrome and SIDS by combined modulation of Nav1.5 and Kv4.3 channel currents. Heart Rhythm 2012; 9: 760-769.

$18 \mathrm{Hu} \mathrm{D}$, Barajas-Martinez H, Burashnikov E et al: A mutation in the beta 3 subunit of the cardiac sodium channel associated with Brugada ECG phenotype. Circ Cardiovasc Genet 2009; 2: 270-278.

19 Olesen MS, Jespersen T, Nielsen JB et al: Mutations in sodium channel $\beta$-subunit SCN3B are associated with early-onset lone atrial fibrillation. Cardiovasc Res 2011; 89: 786-793.

20 Olesen MS, Jensen NF, Holst AG et al: A novel nonsense variant in Nav1.5 cofactor MOG1 eliminates its sodium current increasing effect and may increase the risk of arrhythmias. Can J Cardiol 2011; 27: 523.e17-523.e23.

21 Kattygnarath D, Maugenre S, Neyroud N et al: MOG1: a new susceptibility gene for Brugada syndrome. Circ Cardiovasc Genet 2011; 4: 261-268.

22 Delaney JT, Muhammad R, Blair MA et al: A KCNJ8 mutation associated with early repolarization and atrial fibrillation. Europace 2012; 14: 1428-1432.

23 Medeiros-Domingo A, Tan B-H, Crotti L et al: Gain-of-function mutation S422L in the KCNJ8-encoded cardiac K(ATP) channel Kir6.1 as a pathogenic substrate for J-wave syndromes. Heart Rhythm 2010; 7: 1466-1471.

24 Shinlapawittayatorn K, Dudash LA, Du XX et al: A novel strategy using cardiac sodium channel polymorphic fragments to rescue trafficking-deficient SCN5A mutations. Circ Cardiovasc Genet 2011; 4: 500-509.

25 Poelzing S, Forleo C, Samodell M et al: SCN5A polymorphism restores trafficking of a Brugada syndrome mutation on a separate gene. Circulation 2006; 114: 368-376.

26 Chen L, Zhang W, Fang $C$ et al: Polymorphism H558R in the human cardiac sodium channel SCN5A gene is associated with atrial fibrillation. J Int Med Res 2011; 39: 1908-1916.

27 Bezzina CR, Barc J, Mizusawa Y et al: Common variants at SCN5A-SCN10A and HEY2 are associated with Brugada syndrome, a rare disease with high risk of sudden cardiac death. Nat Genet 2013; 45: 1044-1049.

28 Antzelevitch C, Brugada P, Borggrefe M et al: Brugada syndrome: report of the second consensus conference: endorsed by the Heart Rhythm Society and the European Heart Rhythm Association. Circulation 2005; 111: 659-670.

29 Christophersen IE, Olesen MS, Liang B et al: Genetic variation in KCNA5: impact on the atrial-specific potassium current IKur in patients with lone atrial fibrillation. Eur Heart J 2013; 34: 1517-1525.
30 Sajadieh A, Nielsen OW, Rasmussen V, Hein HO, Hansen JF: Prevalence and prognostic significance of daily-life silent myocardial ischaemia in middle-aged and elderly subjects with no apparent heart disease. Eur Heart J 2005; 26: 1402-1409.

31 Olesen MS, Jabbari J, Holst AG et al: Screening of KCNN3 in patients with early-onset Ione atrial fibrillation. Europace 2011; 13: 963-967.

32 Pfeufer A, van Noord C, Marciante KD et al: Genome-wide association study of PR interval. Nat Genet 2010; 42: 153-159.

33 Sotoodehnia N, Isaacs A, de Bakker PIW et al: Common variants in 22 loci are associated with QRS duration and cardiac ventricular conduction. Nat Genet 2010; 42: 1068-1076.

34 Ritchie MD, Denny JC, Zuvich RL et al: Genome- and phenome-wide analyses of cardiac conduction identifies markers of arrhythmia risk. Circulation 2013; 127: 1377-1385.

35 Yang T, Atack TC, Abraham RL, Darbar D, Roden DM: Abstract 16237: striking electrophysiologic differences between cardiac sodium channel isoforms SCN1OA and SCN5A. Circulation 2011 Abstract.

36 Yang T, Atack TC, Stroud DM, Zhang W, Hall L, Roden DM: Blocking Scn10a channels in heart reduces late sodium current and is antiarrhythmic. Circ Res 2012; 111: 322-332.

37 Verkerk AO, Remme CA, Schumacher CA et al: Functional Nav1.8 channels in intracardiac neurons: the link between SCN10A and cardiac electrophysiology. Circ Res 2012; 111: 333-343.

38 Van den Boogaard M, Wong LYE, Tessadori F et al: Genetic variation in T-box binding element functionally affects SCN5A/SCN10A enhancer. J Clin Invest 2012; 122 . 2519-2530.

39 Derangeon M, Montnach J, Baró I, Charpentier F: Mouse models of SCN5A-related cardiac arrhythmias. Front Physiol 2012; 3: 210.

40 Pazoki R, de Jong JSSG, Marsman RF et al: SNPs identified as modulators of ECG traits in the general population do not markedly affect ECG traits during acute myocardial infarction nor ventricular fibrillation risk in this condition. PLoS One 2013; 8: e57216.

41 Fischer A, Gessler M: Hey genes in cardiovascular development. Trends Cardiovasc Med 2003; 13: 221-226.

42 Kokubo H, Miyagawa-Tomita S, Tomimatsu H et al: Targeted disruption of hesr2 results in atrioventricular valve anomalies that lead to heart dysfunction. Circ Res 2004; 95 : 540-547.

43 Xin M, Small EM, van Rooij E et al: Essential roles of the bHLH transcription factor Hrt2 in repression of atrial gene expression and maintenance of postnatal cardiac function. Proc Natl Acad Sci USA 2007; 104: 7975-7980.

44 Sakata Y, Kamei CN, Nakagami H, Bronson R, Liao JK, Chin MT: Ventricular septal defect and cardiomyopathy in mice lacking the transcription factor CHF1/Hey2. Proc Natl Acad Sci USA. 2002; 99: 16197-16202.

45 Koibuchi N, Chin MT: CHF1/Hey2 plays a pivotal role in left ventricular maturation through suppression of ectopic atrial gene expression. Circ Res 2007; 100: 850-855.

46 Fischer A, Klattig J, Kneitz B et al: Hey basic helix-loop-helix transcription factors are repressors of GATA4 and GATA6 and restrict expression of the GATA target gene ANF in fetal hearts. Mol Cell Biol 2005; 25: 8960-8970.

47 Gollob MH, Jones DL, Krahn AD et al: Somatic mutations in the connexin 40 gene (GJA5) in atrial fibrillation. New Engl J Med 2006; 354: 2677-2688.

48 Abraham RL, Yang T, Blair M, Roden DM, Darbar D: Augmented potassium current is a shared phenotype for two genetic defects associated with familial atrial fibrillation. J Mol Cell Cardiol 2010; 48: 181-190.

49 Tan N, Chung MK, Smith JD et al: Weighted gene coexpression network analysis of human left atrial tissue identifies gene modules associated with atrial fibrillation. Circ Cardiovasc Genet 2013; 6: 362-371.

50 Christophersen IE, Holmegard HN, Jabbari J et al: Rare variants in GJA5 are associated with early-onset lone atrial fibrillation. Can J Cardiol 2013; 29: 111-116. 